\title{
Orléans et le culte public de ses saints fondateurs
}

\section{Françoise Michaud-Fréjaville}

\section{OpenEdition}

Journals

Édition électronique

URL : https://journals.openedition.org/crm/727

DOI : $10.4000 / \mathrm{crm} .727$

ISSN : 1955-2424

Éditeur

Honoré Champion

Édition imprimée

Date de publication : 1 juin 2005

Pagination : 131-141

ISSN : 1272-9752

\section{Référence électronique}

Françoise Michaud-Fréjaville, "Orléans et le culte public de ses saints fondateurs », Cahiers de recherches médiévales [En ligne], 12 spécial | 2005, mis en ligne le 28 juin 2008, consulté le 15 décembre 2022. URL : http://journals.openedition.org/crm/727 ; DOI : https://doi.org/10.4000/crm 727 


\title{
酸M
}

\author{
$-11-$ \\ Orléans et le culte public de ses saints fondateurs
}

La cité d'Orléans n'était encore que récemment convertie au christianisme quand, ayant devant la menace des Huns remis son sort terrestre entre les mains de son évêque Aignan, elle reçut de lui une nouvelle vie'. Les premiers responsables chrétiens de la cité n'ont pas laissé de vraies traces: les historiens du $\mathrm{XX}^{\mathrm{e}}$ siècle finissant hésitent toujours sur les quatre premiers noms des évêques, nuancent les actions qui leur sont attribuées, renoncent à qualifier leur rôle exact, doutent de l'emplacement de leur lieu d'exercice et plus encore de leur première sépulture. Il n'est pas de mon sujet d'apporter en cela de quelconques révélations. Il y a déjà plus de vingt ans, Jean-Marie Berland et Geneviève Renaud on fait un point qui me semble suffisant, dans ses hésitations et ses précisions ${ }^{2}$, à peine modifié dans les publications les plus récentes ${ }^{3}$. Ce qui va être mon propos est le souvenir et la vénération publics accordés, jusqu'à nos jours, aux saints considérés comme les «pères » de la communauté des habitants de la ville.

Il est incontestable qu'Aignan, moins d'une génération après sa mort qualifié de «saint, de très grand et de parfait pontife» par Sidoine Apollinaire ${ }^{4}$, est le pivot de la création par laquelle la mémoire religieuse du clergé et des fidèles orléanais a été façonnée pour des siècles, oserai-je dire allaitée, à partir de la rédaction des différentes hagiographies dont les rédactions semblent s'étaler entre les $\mathrm{VI}^{\mathrm{e}}$ et $\mathrm{XI}^{\mathrm{e}}$ siècles. Je résume ce qui devint la suite logique d'événements censés être connus de tous encore mille ans après : Aignan, successeur d'Euverte, le bâtisseur de la cathédrale Sainte-Croix, et choisi par lui, alla chercher du secours en Arles contre Attila, il guérit (et convertit ?) Mamert à Vienne, il obtint l'aide des pouvoirs officiels impériaux romains: Aetius et ses alliés Wisigoths et Francs arrivèrent à temps pour aider l'évêque à chasser les Huns ${ }^{5}$. La prospérité revint très vite au bord de la Loire.

\footnotetext{
${ }^{1}$ Le 14 juin 451, selon la tradition.

${ }^{2} \mathrm{~J}$.-M. Berland, «Les origines de l'Église d'Orléans (IV ${ }^{\mathrm{e}}-\mathrm{VII}{ }^{\mathrm{e}}$ siècles) », Bulletin de la Société Archéologique et Historique de l'Orléanais, 1978, n 49, p. 19-78; G. Renaud, "Saint Aignan et sa légende, les vies et les miracles ", ibid., p. 83-109. On consultera également Histoire d'Orléans et de son terroir, J. Debal dir., Roanne, 1983, t. I, p. 163-183.

${ }^{3}$ Topographie chrétienne des cités de la Gaule des origines au milieu du VIII siècle, Province ecclésiastique de Sens, J.-C. Picard dir., Paris, 1992, la partie sur Orléans : p. 81-96.

${ }^{4}$ Sidoine Apollinaire, Lettres, éd. A. Loyen, Paris, 1970, t. III, liv. VIII, XV, p. 126-127, lettre à l'évêque Prosper (vers 479). Les qualificatifs donnés à Aignan sont sanctus, maximus, consummatissimus.

${ }^{5}$ On sait que la Vita I (rédigée après 474 et avant 530) enregistre la fondation par Euverte d'un grand édifice non-dénommé, mais dit que plusieurs évêques s'étaient succédés entre Euverte et Aignan; elle rappelle que la ville dut bien ouvrir ses portes à Attila mais n'eut pas le temps d'être pillée (cf. A. Loyen, «Le rôle de saint Aignan dans la délivrance d'Orléans », Comptesrendus de l'Académie des Inscriptions et Belles-Lettres, 1969, $1^{\mathrm{er}}$ trimestre, p. 64-74). Grégoire de Tours préfère choisir une version qui fait encore résister la ville au bord de l'écroulement (Histoire des Francs, II, VII, cette partie fut rédigée entre 543 et 584).
}

Cahiers de Recherches Médiévales (XII -XV s.), 12spé, 2005 
À partir de ces faits et des trois personnages (Aignan, Euverte et Mamert) qu'ils évoquent, je voudrais réfléchir aux manifestations publiques de commémoration dont ils furent couramment honorés au cours des siècles orléanais. Thomas Head a longuement étudié, en prenant justement l'exemple de notre région, comment les héros chrétiens de la résistance aux barbares acculturèrent ces derniers ${ }^{6}:$ le Franc Gontran se rendant en procession en 585 dans les lieux de culte orléanais en compagnie de Grégoire de Tours et d'une série d'évêques est une des plus anciennes manifestations de l'alliance, parfois houleuse, des conquérants avec les anciennes élites sociales et religieuses d'une ère romaine désormais révolue? ${ }^{7}$ Cet épisode me paraît aussi une étape essentielle dans la familiarité des habitants de la ville avec les restes mortuaires de leurs saints et avec leurs dirigeants. La visite de Louis le Pieux en 814 semble un doublet, significatif de la volonté carolingienne de se placer dans les pas de la dynastie précédente ${ }^{8}$. Les efforts des religieux pour ensuite fixer par les textes de diverses vitce les actions humaines et thaumaturgiques n'ont fait que donner une forme littéraire, souvent tardive, aux certitudes des fidèles à l'égard des pouvoirs protecteurs des saints patrons.

Les trois saints principaux de la tradition épiscopale orléanaise doivent être examinés de près, sans nous attacher cependant à tous les menus détails historicolégendaires auxquels aboutissent au tournant du premier millénaire les diverses relations écrites. Nous y trouvons un bâtisseur de la maison qui accueille l'Ecclesia, consécrateur des clercs, portant le calice du sacrifice que Dieu lui-même a consacrée, le prêtre Euverte. Nous rencontrons un défenseur actif, un faiseur de paix qui pour cela sait prévoir la guerre, se dépenser en ambassades, élever des murailles réelles (celle du Bas-Empire) ou fictives (le brouillard à la mi-juin qui va empêcher l'assaut des Huns), un guerrier, ainsi apparaît Euverte. Enfin, sur sa route, le chef de guerre élu et béni par le prêtre nous présente un laïc guéri des vieux démons et converti à la vie juste: l'œuvre fondamentale du miraculé de Vienne sera désormais d'attirer la grâce de Dieu sur les fruits de la terre par la pratique des Rogations, voici le producteur Mamert ${ }^{10}$. On reconnaîtra sans peine, et sans que je croie avoir forcé le trait, les

${ }^{6} \mathrm{~T}$. Head, Hagiography and the cult of saints. The diocese of Orléans, 800-1200, Cambridge, 1990. L'auteur s'est surtout attaché aux relations entre les communautés monastiques rédactrices des Vitce avec les pouvoirs divers, et à l'usage qu'elles firent de ces textes pour conserver ce qu'elles considéraient comme leurs libertés.

${ }^{7}$ Grégoire de Tours, op. cit., VIII, 1. Lors de son séjour lors de la Saint-Martin d'été, les 4 et 5 juillet 585 , Gontran eut plusieurs altercations avec ses évêques, dont il souligna le double langage et les fidélités douteuses.

${ }^{8}$ Ermold le Noir, Poème sur Louis le Pieux, éd. E. Faral, Paris, 1932 (Classiques de l'histoire de France), t. II, p. 62.

${ }^{9}$ Selon la Vita II de saint Euverte (IX ${ }^{\mathrm{e}}$ siècle), lors de la dédicace de la nouvelle église d'Orléans, une main sortit des nuées au moment de la consécration et une voix aurait dit: hoc templum Dei manu consecratum. Le calice que saint Euverte aurait tenu ce jour insigne figure sur la liste des reliques orléanaises du Codex callixtinus, plus connu aujourd'hui comme le Guide du pèlerin de saint Jacques de Compostelle.

${ }^{10}$ On peut se préoccuper de trouver très tôt $\left(\right.$ Vita Aniani $\left.I^{a}\right)$ la mention d'un retour fort rapide (trois ans) de la prospérité orléanaise après 451. L'évêque Prosper des années 470, correspondant attesté de Sidoine Apollinaire, fut peut-être le déclencheur de la légende de la guérison du saint évêque de Vienne par Aignan, épisode historicisé par l'accueil et la conservation à la cathédrale de reliques de Mamert. Mamert fut évêque de 463 à 467. L'institution des proces- 
trois ordres que décrivaient déjà les penseurs médiévaux ${ }^{11}$ et que nos maîtres, à la suite des mythologues de la trifonctionnalité, ont longuement et finement analysés ${ }^{12}$.

À la limite, qu'auraient importé les fragments de corps saints et les reliques diverses qui occupèrent avant le $\mathrm{XI}^{\mathrm{e}}$ siècle les églises basses des grands sanctuaires urbains avant d'être exposés sur les autels ou conservés dans les trésors ${ }^{13}$, si les trois protecteurs efficaces de la cité n'y étaient effectivement présents. Les processions dont les rites se déroulaient plusieurs fois par an étaient les occasions de renouer, de renforcer les liens entre le peuple et ses saints. On ne veut pas refaire ici l'historique ni le détail de ces manifestations ${ }^{14}$, mais il me paraît nécessaire pour le sujet de ce colloque de relever la place tenue par les saints fondateurs - à côté des autres - dans ces cérémonies de cohésion urbaine. J'ai fait cette petite étude pour le premier quart $\mathrm{du} \mathrm{XV}^{\mathrm{e}}$ siècle, période, on le sait, particulièrement difficile dans la partie médiane de la vallée de la Loire, un des nœuds de la guerre larvée puis civile qui fit rage entre les Bourguignons et les Armagnacs dont on n'ignore pas que, pour ces derniers, il s'agit bien des ducs d'Orléans et de leur clientèle. Les sources municipales orléanaises permettent, en dépit de la destruction quasi totale des archives anciennes de la ville en 1940, de suivre de près les manifestations processionnelles de la cité pendant plusieurs dizaines d'années.

Entre 1401 et 1430 , cent onze processions sont rappelées à notre mémoire par les comptes de la ville. Hélas pour nous, la plupart n'énumèrent pas les différentes reliques exposées aux fidèles; nous en avons cependant cinquante qui permettent de relever la place tenue par les diverses châsses ou groupes de châsses portées au long des rues.

La fierté des ancêtres épiscopaux dut-elle en souffrir, c'est Mamert qui vient en tête, non seulement à l'occasion des Rogations, mais à chaque fois que la météorologie ou la guerre rendent les ressources du sol et les passages de marchandises aléatoires. L'ange noir de la famine est celui qui sème la première terreur. C'est presque dans une procession sur deux que Mamert est appelé pour lui faire face (44\% des manifestations). Puis-je rappeler à cette occasion que la peste, en général, annule toutes les processions : deux pages des Très riches Heures du Duc de Berry nous ramènent en un temps où les flèches de la mort subite sont imparables : la procession de la grande litanie de saint Grégoire le Grand y est décimée dans sa déambulation sacrée, les reliques tombant des mains d'un prêtre qui s'effondre, un moine ne passe pas le seuil de la ville et une femme se pâme devant ses enfants alors même

sions des rogations pour obtenir des récoltes abondantes ne devint officielle et fixée aux trois jours avant l'Ascension que sous le pontificat de Léon III $(† 816)$.

${ }^{11}$ «Les uns prient, d'autres combattent et d'autres encore travaillent... ».

${ }^{12} \mathrm{G}$. Duby, Les trois ordres ou l'imaginaire du féodalisme, Paris, Gallimard, 1978; voir les limites pertinentes qu'indique le compte-rendu de R. Fossier (Revue historique, 1980, $\mathrm{n}^{\circ} 533$, p. 296-298).

${ }^{13}$ On vénérait les fragments des corps des saints Aignan, Euverte, Mamert, Euspice, Monitor, Flou, Baudel, Sublius, Savinien et Potentien, Avit (ou Ay), Samson, Paul ermite, Benoît, Victor, Marcel, Evroul, sainte Agie et les objets sacrés qu'étaient un fragment de la Vraie Croix, le calice de saint Euverte et la patène de la Cène.

${ }^{14} \mathrm{~F}$. Michaud-Fréjaville, «Les processions à Orléans au $\mathrm{XV}^{\mathrm{e}}$ siècle », Revue Mabillon, nouvelle série, 6 (t. 67), 1995, p. 203-223, réimpr. supra, article n 10. 
que, paradoxe, l'archange de la mort rengaine son épée sanglante ${ }^{15}$. Après Mamert, et le suivant de très près, vient Aignan, ce qui peut quand même nous conforter eu égard au sujet de la présente rencontre sur le thème des églises locales. En cette période de grands troubles et de menace de sièges, on préférait sortir le corps tout entier (ou sa plus grande partie) plutôt que le seul chef (c'est-à-dire des os du crâne). Vénérée et offerte à la dévotion à des fins propitiatoires à l'égal du corps de saint Aignan, ensuite viennent les reliques de la Vraie croix. Il me semble que cette place en retrait est un signe, non d'une déviation de la religion chrétienne vers une sorte de polythéisme au bénéfice des saints locaux mais bien d'une forme de réserve cléricale devant des restes de provenances seulement quasi certaines, de tradition sincère puisque - disait-on - d'époque constantinienne, mais moins directement efficaces que la sanctification des os autochtones. Enfin et loin derrière, viennent les reliques de saint Euverte dont nous n'avons d'attestations que dans $16 \%$ des cas ${ }^{16}$.

En 1501 encore, il fallait soixante-douze porteurs pour permettre le déplacement général de ces châsses, ce qui laisse deviner le déroulement solennel de ces cérémonies. Le nombre des brancardiers permet, par un autre biais, de mesurer la popularité des saints par l'ampleur des reliquaires qui ont, à la fin du Moyen Âge, souvent été rénovés ou agrandis : les saints Aignan, Euverte et Mamert ont chacun besoin de deux série de porteurs, seize pour Euverte, dix pour Mamert, douze pour Aignan, à chaque fois huit pour le «corps » et respectivement huit, quatre et deux pour le chef des saints. Les trois patres patrice ont donc conservé leur prééminence sur les autres corps saints, un millénaire après la reconnaissance par la cité de leur caractère protecteur contre les ennemis militaires ou météorologiques, encore faudrait-il savoir si leurs rôles étaient encore perçus de la même façon.

Le peuple chrétien est, sans jeu de mot, un peuple fidèle : la commémoration de la délivrance de la ville par Jeanne d'Arc et les troupes royales n'a absolument pas envoyé immédiatement les grands ancêtres aux oubliettes. La commémoration annuelle fut toujours placée sous le signe des saints traditionnels : dans cette cérémonie d'action de grâce, la procession était toujours celle des corps conservés dans les châsses des églises de la cité et tout particulièrement celles de saint Euverte et de saint Aignan car, dit le texte du récit de l'établissement de la fête, des «Anglois estant pour lors au dit siège [assuraient] avoir veu deux prélats en habit pontifical aller et venir en cheminant par sus les dits murs de la dicte ville d'Orléans, ainsi

${ }^{15}$ Les Très riches Heures, fol. 71v.-72 : c'est le moment où, selon la Légende dorée, au sommet du mausolée d'Hadrien (château Saint-Ange), saint Michel apparut au pape Grégoire, vision qui annonçait la fin de l'épidémie (590).

\begin{tabular}{|l|l|l|}
\hline $\begin{array}{l}\text { Reliques nommément désignées par les comptes } \\
\text { de ville }\end{array}$ & $\begin{array}{l}\text { Nombre de sorties } \\
\text { (sur 50) }\end{array}$ & $\%$ \\
\hline Saint Mamert & 22 & 44 \\
\hline Vraie Croix & 13 & 26 \\
\hline Chef saint Aignan & 9 & 18 \\
\hline Corps saint Aignan & 13 & 26 \\
\hline Saint Euverte & 8 & 16 \\
\hline
\end{tabular}

LES RELIQUES DES SAINTS DANS LES PROCESSIONS ORLÉANAISES 1400-1430

(N. B. Des processions comportent plusieurs reliques, voire toutes, les deux reliquaires de saint Aignan n'ont été sortis que deux fois ensemble, et six fois les trois saints). 
toutes fois ont esté gardes et protecteurs, les dits saints Euverte et monseigneur saint Aignan, de ladite ville au temps que vindrent devant elle mecreans ${ }^{17}$. Le rappel est évident de la scène décrite par Grégoire de Tours où Aignan, du haut des remparts, guettait les troupes d'Aétius et ses alliées, surveillait le camp d'Attila et le noyait dans la purée de pois. Qui mieux que les vaincus pouvaient certifier que leur défaite était d'origine surnaturelle ! Dans un premier temps, le miracle de la levée du siège de 1429 fut véritablement interprété comme un doublet de celui de 451. Quand en 1468 le jeune Éloi d'Amerval dirigeait la maîtrise des enfants de chœur d'Orléans, l'hymne qu'on lui attribue et dont on a gardé le souvenir s'adresse d'abord aux saints évêques : "Chacun an y faisons belle procession /en chantant chants de paix et motets gracieux/ à benoist saint Aignan tant digne et précieux/ o saint Euverte aussi, nos patrons glorieux». Nous ne devons donc pas être surpris de la hiérarchie des porteurs de brancard de 1501.

La place accordée à la Pucelle dans l'imaginaire et le cérémonial orléanais actuels ne doit ainsi pas nous aveugler; autrefois elle n'avait pas encore envahi les lieux au point de ravaler les glorieux pères au rang d'utilités : la «bannière de la ville», portée lors de la fête du 8 mai du XVI ${ }^{\mathrm{e}}$ au XVIII ${ }^{\mathrm{e}}$ siècle, en est une preuve de plus. C'est un objet mal connu, mais qui devrait inciter fortement les Orléanais à la réflexion historique ${ }^{18}$. La bannière insiste sur le caractère civique particulier du monument à la Pucelle qui se trouvait sur le pont. Ce n'est pas ici le lieu d'étudier en elle-même cette représentation - ou plutôt interprétation - de l'ensemble sculpté commémoratif $^{19}$, mais seulement d'en dégager ce qui importe à notre sujet. Jeanne et son vis-à-vis, le duc d'Orléans et non pas le roi, sont à genoux devant la Vierge et l'enfant, dans la disposition qui se présentait aux passants, Jeanne à la gauche de la Vierge, et le seigneur de la ville, donc le duc, à sa droite, selon une hiérarchie toute humaine. La grande différence entre la bannière et la sculpture tient à ce que les orants soient présentés, à la manière traditionnelle des donateurs d'œuvres cultuelles, par deux évêques en grands habits pontificaux, chape, mitre et $\operatorname{crosse}^{20}$ : Aignan derrière le duc, Euverte derrière Jeanne. C'est au duc que s'adresse Jésus en lui touchant la main, geste aristocratique d'honneur particulier, tout en répondant aux oraisons des deux prélats et surtout à la prière des orants inscrite sur un cartouche placé devant le prince : «Seigneur donne-moi un signe favorable» par le phylactère : «je dégainerai mon glaive et que ma main les détruise». Que le contexte soit celui de l'invasion des Huns, de la guerre de Cent ans ou celui des guerres de religion lors de la commande de l'objet, comme semble le laisser penser l'envers de la bannière, les patres patrice dominent la situation : la prière commune de Jeanne et du duc, et

${ }^{17} \mathrm{~A}$. Salmon, "Chronique du siège d'Orléans et de l'établissement de la fête du 8 mai », Bibliothèque de l'École des Chartes, t. 8, 1846, p. 500 et suiv.

${ }^{18}$ Orléans, Musée historique de l'Orléanais, inv. A 8345. J. de la Martinière, «Trois bannières de la ville d'Orléans », Bulletin de la Société Archéologique et Historique de l'Orléanais, 1933 , p. 262 et suiv.

${ }^{19} \mathrm{~F}$. Michaud-Fréjaville, «Images de Jeanne d'Arc, de l'orante à la sainte », Images de Jeanne d'Arc, Actes du colloque de Rouen 25-27 mai 1999, J. Maurice et D. Couty dir., Paris, 2000, p. 243-251, réimpr. infra, article 21.

${ }^{20}$ L'instigateur du monument fut Aignan II de Saint-Mesmin († 1498), voir Mgr P. Brun, «Le premier monument à Jeanne d'Arc sur l'ancien pont d'Orléans ", Les Dossiers de l'archéologie, 1979, n 34, p. 71-76. 
celle du corps de ville et de l'Église sur l'autre face de la bannière, passe d'abord par eux pour être exaucée.

Les siècles passent et le 8 mai, perdant son vieux nom du «Lèvement des Tourelles », devient la «Fête de la ville » ou la «Fête de la Pucelle» (marque déjà du caractère personnalisé de la cérémonie), mais les protecteurs demeurent, médiateurs de la prière collective des Orléanais. Les reliques, moins nombreuses peut-être en raison des destructions des guerres protestantes, sont toujours portées par les voies de la cité de la cathédrale aux Augustins, par le pont qu'avait repris l'armée de Jeanne en 1429. La forme de la ville peut changer, les évêques fondateurs tiennent bon. En 1772 on jeta un pont neuf, qui rendit nécessaire la définition d'un nouvel itinéraire pour les cérémonies du mois de mai : le petit livret d'explication proposé par Louis Sextius de Jarente va bien au-delà de l'ordre du cortège car il donne également le programme de la liturgie de déambulation ${ }^{21}$. On ne s'étonnera pas : après le chant de départ vient le répons de saint Mamert ${ }^{22}$, auquel en écho succède celui de saint Aignan ${ }^{23}$ et tous deux mènent le peuple jusqu'au milieu du pont «royal », d'où l'on peut contempler toute la ville. Jeanne n'est pas directement évoquée, elle se trouve cependant honorée par son double biblique qu'est Déborah, la prophétesse : «Alors a surgi la mère en Israël. / Le Seigneur fit une guerre nouvelle /et son armée a abattu les portes $~^{24}$. Enfin la procession du retour entre dans la cathédrale avec une série d'hymnes à la Sainte Croix : la commémoration d'Euverte, pour être plus discrète, est néanmoins toujours là.

S'agit-il ici d'un état fossilisé de la liturgie, d'un usage pratiquement interne à un groupe clérical en décalage avec le peuple chrétien? Le journal de Sylvain Rousseau, le grainetier du Martroi qui nota fidèlement l'éphéméride de sa ville de 1780 à 1805 , signale scrupuleusement le temps qu'il a fait pour chacun des 8 mai d'Ancien Régime et la manière dont la procession s'était déroulée sous un ciel qui ne fut pas toujours clément ${ }^{25}$. «Le 8 courant, il n'a point tombé d'eau, ce qui a doné occasion de faire la procession de la Pucelle ${ }^{26}{ }^{2}$. L'habitude rend peut-être moins vigilant aux antiennes, mais si l'usage avait été rompu, si quelque nouveauté s'était insérée, immédiatement nous en aurions eu un écho, comme l'initiative du duc d'Orléans de doter une rosière à partir du 8 mai 1786. Sylvain Rousseau, par ailleurs, utilise presque systématiquement pour le jour de l'anniversaire de la déli-

\footnotetext{
${ }^{21}$ Ordre de la procession générale qui se fait tous les ans le 8 mai, en action de grâce à Dieu pour la délivrance de la ville d'Orléans du siège des Anglois, par l'entremise de Jeanne d'Arc, communément appelée la Pucelle d'Orléans, Orléans, chez J. M. Rouzeau-Montaut, 1790. L'évêque Alexandre Jarente Sénas d'Orgeval (év. 1788 - 21 nov. 1793) a juré la constitution civile du clergé dès le 30 janvier 1791.

${ }^{22}$ Ecce sacerdos magnus qui in diebus suis placuit Deo et inventus est justus. Et in tempore iracundice factus est reconciliatio, Alleluia.

${ }^{23}[\ldots]$ Hic est fratrum amator, et populi Israel : hic est qui multum orat pro populo et universa civitate ...

${ }^{24}$ Juges, 5, 7-8 : Donec surgeret mater in Israel. Nova bella elegit Dominus et portes hostium ipse subvertit.

${ }^{25}$ Le Journal de Sylvain Rousseau est conservé par la Société Archéologique et Historique de l'Orléanais. Pour les pages du Journal concernant les fêtes du 8 mai année par année, F. Michaud-Fréjaville, «Les fêtes du 8 mai dans le journal de Sylvain Rousseau, 1770-1805 », Bulletin de la Société des Amis du Centre Jeanne d'Arc, 1990, n 14, p. 35-37.

${ }^{26} \mathrm{~S}$. Rousseau, Journal, t. I, 1782, p. 125.
} 
vrance de la cité le terme de «feste de la ville, jour de la Pucelle» (1770, 1776. 1779) : Jeanne est passée au premier plan.

Ce qui a véritablement cassé une première fois le lien entre la ville et ses pères fondateurs de l'aube des temps chrétiens fut la Révolution. On avait choisi le 9 mai 1790 pour célébrer la fête de la fédération aux Quatre-Vents d'Olivet. La procession du 8 , sous une pluie continue, fut surtout celle des volontaires se préparant à la revue ${ }^{27}$. Après 1793, la dispersion des reliques et l'interdiction des processions religieuses sonnèrent la fin du jour de la Pucelle. Les liens de la mission de Jeanne avec le sacre et la royauté rendaient alors impossible toute républicanisation de la fête de la ville. En 1793 tout fut interdit; le 9 juin, on célébra dans la cathédrale le culte de l'Être suprême. Les fêtes révolutionnaires des années 1793-1794, nombreuses, prirent dans les rues la place des processions, y compris dans leur traversée du fleuve $^{28}$, sans que nous sachions très bien quelle importance réelle leur accorder dans le maintien orléanais d'un sentiment d'appartenance municipale.

Or très tôt la conjoncture politique permit la réintroduction d'un culte chrétien de cohésion civique avec la promotion non encore d'une sainte mais d'une héroïne du peuple, Jeanne d'Arc. La lutte contre l'Angleterre qui devait mobiliser les énergies patriotiques, ajoutée au succès de la pièce de Schiller qui touchait les âmes sensibles du préromantisme, permit d'abord au premier consul, soucieux de faire avancer la réconciliation religieuse à laquelle l'évêque Étienne Alexandre Bernier, négociateur du Concordat, avait déjà activement contribué, d'autoriser la réintroduction de la procession du 8 mai $^{29}$. "Nous dirons qu'il n'y a jamais eu rien de plus superbe en fait de procession. Monseigneur notre évêque y estoit bien accompagné de son clergé », nous rapporte dans l'enthousiasme le brave Sylvain Rousseau au 8 mai de l'année $1803^{30}$. Cependant il manque dans son récit la longue théorie des châsses pesant aux bras des porteurs. Les saints évêques sont encore absents des commémorations citoyennes. Ce sera chose réparée dès le mois suivant, comme si une fête de la ville sans ses patrons était décidément chose impossible. Les reliques de saint Aignan, cachées pendant toute la période révolutionnaire rue Sainte-Anne dans la maison «des Oves », furent solennellement replacées dans leur église en juin $1803^{31}$. La sollicitude du premier consul et les vœux du clergé et de la ville se retrouvèrent l'année suivante quand fut inaugurée la statue de Gois place du Martroi et que la journée se finit par un feu d'artifice, «le tout en l'honneur de la Pucelle d'Orleans mise ou posee sur son pied d'estalle depuis deux jours, visible le huit, jour

${ }^{27}$ Ibid., t. II, p. 99.

${ }^{28} \mathrm{D}$. Lottin, Recherches historiques sur la ville d'Orléans, Orléans, 1836-1845, t. II, 2, 3, 4. Les trajets ont été cartographiés par Jean Vassort (Histoire d'Orléans et de son terroir, op. cit., t. II, p. 172-173).

${ }^{29}$ "L'illustre Jeanne d'Arc a prouvé qu'il n'est point de miracle que le génie français ne puisse opérer », avait noté le Premier Consul en marge de la demande de la municipalité et du préfet Maret. Ce document est conservé au Centre Jeanne d'Arc d'Orléans.

${ }^{30}$ S. Rousseau, Journal, t. II, p. 367.

${ }^{31}$ C.-F. Vergnaud-Romagnési, Orléans et ses environs, Guide historique, statistique et commercial..., Orléans, Gatineau, 1836, p. 42 : « les reliques de saint Aignan, brûlées puis retrouvées depuis 1562, disparurent en 1793 ; reconnues en 1803 par l'évêque Bernier, elles sont contenues dans une châsse... ». Sur le lieu de leur abri révolutionnaire : J. Vassort, Histoire d'Orléans, op. cit., p. 189. 
de la feste. Amen $»^{32}$. Il ne s'agissait cependant pas d'une restauration totalement à l'identique. Sylvain Rousseau a noté, non sans une pointe d'humour, voire de nostalgique réalisme, au 8 mai 1805 : «sur le midi, la procession est sortie de ste Croix, accompagnée des pretres et viquaires de toutes les paroisses voisines de la ville, [ils] sont allés a st Marceau ainsi qu'il est d'usage, autrefois on allait aux Augustins mais aujourd'hui c'est une raffinerie [de sucre] ${ }^{33}$.

On sait que la célébration de la fête acceptée par le premier consul, a perduré, avec quelques à-coups, jusqu'à nos jours ${ }^{34}$. Des coutumes nouvelles se sont peu à peu instaurées : disparu le «puceau » d'ancien régime, on superposa au $\mathrm{XX}^{\mathrm{e}}$ siècle la présence d'une jeune fille en armure de l'âge de Jeanne, mais Orléanaise, ce qui peut paraître bien réducteur, avec l'invitation systématique depuis trente ans du président de la République l'année de son élection, et enfin la célébration officielle et entremêlée du 8 mai $1945^{35}$. Mais les saintes reliques ne passent plus aujourd'hui par les rues, alors que le clergé et les «mai » des paroisses défilent encore ${ }^{36}$. La chronologie de la disparition des corps saints mérite de s'y arrêter. En 1855, à l'occasion de l'inauguration de la statue équestre de Foyatier sur la place du Martroi, qui reléguait de l'autre côté de l'eau celle de Gois, démodée par ses panaches et jamais vraiment appréciée ${ }^{37}$, le programme du défilé prévoyait, aux $18^{\mathrm{e}}$ et $19^{\mathrm{e}}$ rangs du cortège, les «bannières de saint Euverte et de saint Aignan», puis au $29^{\mathrm{e}}$ rang la croix et le clergé des paroisses de la ville, au $30^{\mathrm{e}}$ les «châsses des saints portées par les élèves du grand séminaire et accompagnées de seize torches aux armes de la ville», puis vient le chapitre de la cathédrale, les prélats et enfin, antépénultièmes et $34^{\mathrm{e}}$ du défilé, les corps constitués et les invités de la ville ${ }^{38}$. On peut noter que saint Mamert a disparu

${ }^{32}$ S. Rousseau, Journal, t. III, p. 19. Le principe de l'érection de cette statue avait été adopté dès l'année précédente. Edme Gois (fils) avait obtenu avec elle en 1801 un prix au concours proposé par Lenoir sur le thème de Jeanne d'Arc ; un modèle en avait été exposé le 8 mai 1803. La statue, plusieurs fois déplacée, est désormais à l'emplacement des Tourelles.

${ }^{33}$ S. Rousseau, Journal, t. III, p. 40. A. Prost rappelle que cette année-là un incident eut lieu entre le préfet, le maire et l'évêque, le maire soulignant que «le cérémonial a toujours été réglé par les maires d'Orléans » (A. Prost, «Jeanne à la fête, identité collective et mémoire à Orléans depuis la Révolution française », dans La France démocratique, Mélanges offerts à Maurice Agulhon, Paris, 1998, p. 382).

${ }^{34}$ Une éclipse de la procession traditionnelle eut lieu entre 1831 et 1841 : la commémoration du 8 mai eut alors un caractère religieux dans l'espace clos de la cathédrale et un espace public, sans clergé, par l'honneur de la Garde nationale rendu au buste de Jeanne d'Arc.

${ }^{35}$ Les articles concernant les fêtes d'Orléans depuis la Révolution sont extrêmement nombreux et de valeur très inégale, je renverrai à celui d'Antoine Prost cité note 33, un des plus récents, remarquable.

${ }^{36}$ Les « mai » des paroisses font entrer dans la fête le souvenir des manifestations de jeunesse d'Ancien Régime (Mona Ozouf, «Du mai de liberté à l'arbre de la liberté : symbolisme révolutionnaire et tradition paysanne », Ethnologie française, V, 1975, p. 9-32).

${ }^{37}$ C.-F. Vergnaud-Romagnesi, Orléans et ses environs, op. cit., p. $31:$ : on conserve toujours à Orléans l'espoir de lui élever une statue équestre, de grandeur et de forme et de pose plus satisfaisante (sic)». Rappelons que ce texte est paru en 1836.

${ }^{38}$ Journal du Loiret, XXXVIII ${ }^{\mathrm{e}}$ année, ${ }^{\circ} 46$, mardi 17 avril 1855, p. 1. Lors de ces fêtes, on avait commencé le 7 mai par une cavalcade historique; après le défilé du 8 mai, on termina par l'inauguration de l'Hôtel de ville (ou Hôtel Groslot), «nouvellement restauré» dans l'allure extérieure et la décoration intérieure que nous lui connaissons encore, qui comporte 
nominalement de la célébration. Quinze ans plus tard, l'affiche officielle des fêtes du $440^{\mathrm{e}}$ anniversaire (1869) ne montre pas une enflure exagérée du défilé, mais désormais les préséances ont changé, le civil a pris le pas sur le sacré : les édiles, Eugène Vignat, maire, et ses adjoints, se sont avancés au $20^{\mathrm{e}}$ rang, les châsses des paroisses de la ville suivent trois rangs plus loin, fort près finalement de ce corps de ville qui reste largement fidèle à sa proximité traditionnelle des reliques, au moins au vu et au su de l'ensemble des assistants. Un corps de lanciers précède au $25^{\mathrm{e}}$ rang un second groupe du clergé et de reliques : les châsses de la cathédrale et les évêques. Au tournant $\mathrm{du} \mathrm{XX}^{\mathrm{e}}$ siècle, les cartes postales montrent que le cortège des corps saints était loin de nécessiter les cohortes de porteurs médiévaux: vers 1908, deux suffisaient pour chacun des objets exhibés lors de ce que l'on appelle alors couramment la «procession de Jeanne d'Arc». Répandues à profusion, ces cartes popularisent dans la France entière les solennités de plus en plus consacrées à une héroïne en voie de canonisation. Mais les légendes, si elles précisent parfois les noms ou les fonctions de certaines des personnalités ou ceux des groupes, ne révèlent rien sur les pères fondateurs que l'on continuait à présenter derrière les bannières, anonymes ou presque dans leurs petites boîtes, par les rues en fête.

En 1955, cent ans après Monseigneur Dupanloup, pour une fête qui s'intitule de manière assez désinvolte «Fête Jeanne d'Arc» jusque sur la couverture du programme officiel, les rangs du cortège se sont considérablement accrus, il y eut cette année-là quatre-vingt-sept rangs. Le maire et les corps constitués sont au $68^{\mathrm{e}} \mathrm{rang}$, la "croix du Chapitre suivie des châsses de Sainte-Croix, de Saint-Aignan et SaintEuverte» viennent au $77^{\mathrm{e}}$ rang et l'évêque au $84^{\mathrm{e}_{39}}$. Dix ans plus tard, en 1965, les châsses figurent dans le défilé au $70^{\mathrm{e}}$ rang, immédiatement suivies d'une musique de la $2^{\mathrm{e}}$ région aérienne qui martialise la pieuse déambulation, l'évêque cinq rangs plus loin était talonné par la Fanfare des pieds-blancs des Aydes ${ }^{40}$. Faut-il y voir un brin d'anticléricalisme? C'était cependant l'exacte répétition de l'année précédente ${ }^{41}$, et ne présageait donc pas la véritable mutation de l'année suivante.

En 1966, au cours d'un cortège un peu plus resserré, la partie religieuse du défilé commémoratif du 8 mai voyait disparaître, après cinq cent trente-six ans, l'exposition des châsses des saints protecteurs de la cité devenus d'ailleurs déjà dans les programmes des années précédentes et par la vertu de la typographie les simples objets d'églises de la ville plus ou moins désaffectées ${ }^{42}$. L'hommage du clergé à la Pucelle se réduisait à quatre rangs qui encadraient - à l'époque - l'étendard de Jeanne. Passaient successivement la croix du chapitre, le grand séminaire, le clergé de la ville, l'évêque et les prélats invités. La Fanfare des Pieds-Blancs était remplacée par l'Harmonie Saint-Joseph d'Olivet, au nom plus catholique. L'abandon des reliquaires correspondait, certes, à une sensibilité publique et religieuse très éloignée

dans le salon d'honneur cinq représentations de Jeanne d'Arc, plus quatre des saints fondateurs (chacun a un tondo de vitrail).

${ }^{39}$ Programme officiel des fêtes de 1955, p. 10-12.

${ }^{40}$ Programme officiel des fêtes de Jeanne d'Arc, 1965, p. 18-19.

${ }^{41}$ Programme de la célébration du $535^{\mathrm{e}}$ anniversaire, 1964, p. 6.

${ }^{42}$ Programme officiel des «Fêtes de Jeanne d'Arc, 1966», p. 19. Remplacer l'expression de 1855 «châsses des saints » (avec s minuscule) par châsses de Saint-Aignan, Saint-Euverte etc., montre que typographes et relecteurs confondent désormais le lieu de conservation et les reliques du saint personnage. 
sur les bords de Loire des usages méditerranéens, mais cette prise de distance relève aussi d'un sentiment semble-t-il très net que les saints traditionnels n'avaient plus rien à faire là. Comme s'était perdue la mémoire des Huns, les saints se sont à leur tour évanouis dans la brume. Le prétexte à l'abandon semble avoir été que les porteurs auraient dû être des clercs et qu'ils n'étaient plus assez nombreux. En fait, cette assertion n'a rien de canonique : au Moyen Âge, les porteurs étaient des gens de la ville, purs et simple laïques aux gros bras, indemnisés pour leur tâche de quelques deniers et d'un bon coup à boire dans une taverne des plus prosaïques de la cité ; le rapport des chrétiens et des saints médiévaux avait parfois un caractère moins sacré et plus direct qu'on le pense ${ }^{43}$. L'argument est d'autant plus faible qu'une carte postale montre les grosses moustaches et le chapeau mou d'un laïc dans les brancards (v. 1908). En ce troisième tiers du $\mathrm{XX}^{\mathrm{e}}$ siècle, les grands élèves des établissements religieux de la ville rechignaient sans doute de plus en plus à accomplir un long périple, douloureux aux épaules, pour des objets qui ne provoquaient plus sans doute de marques de vénération et risquaient au contraire de subir de déshonorants quolibets. D'autant que la Pucelle, devenue l'objet d'un culte et unique sujet d'émerveillement des Orléanais, n'avait justement laissé aucune relique, donc aucun reliquaire, à proposer à la vue d'un public qui savait tout sur elle et plus grand chose d'Euverte et d'Aignan ${ }^{44}$.

La promotion de Jeanne, déjà systématiquement appelée la «sainte fille» au $\mathrm{XVII}^{\mathrm{e}}$ siècle dans La Pucelle de Chapelain, et passée au rang des élus depuis la fin du XIX ${ }^{e}$ siècle et la proclamation de sa vénérabilité, ne signifie peut-être pas qu'ait disparu totalement pour les participants et les spectateurs de la fête de la ville la rassurante trifonctionnalité qui pour une bonne part permet encore à nos sociétés de se croire à peu près cohérentes. La canonisation de la martyre en 1920 lui a permis de représenter la fonction sacrée; elle est la patronne de la France, et la médiatrice pour les Orléanais, en lieux et places des anciens évêques. Sa présence en armure dans le cortège et le rappel des étapes de la campagne de la levée du siège la placent très naturellement dans le groupe des guerriers, et la double célébration des victoires des 8 mai 1429 et 1945 n'a fait que renforcer son caractère martial. Et toute cléricalisée qu'elle puisse être, tout engoncée dans sa ferraille, elle est enfin, et c'est sans doute ce qui la rend encore si émouvante et familière pour tous, la paysanne décidée et volontaire de Michelet et de Péguy, le symbole du troisième ordre, le peuple. Sur les affiches, les «fêtes de Jeanne d'Arc, civiles, militaires et religieuses » ne suggèrent rien d'autre.

La cité d'Orléans, ayant perdu peu à peu la familiarité de ses anciens pères fondateurs, n'a pas eu à se réinventer des modèles, Jeanne est devenue la «mère en Orléans », pour paraphraser le cantique de Déborah. L'état de décrépitude dans la-

${ }^{43}$ Arch. Dép. Loiret, CC 646, XXII (compte de la ville, 1406) : [Au valet de la ville, Jacquet Le Prestre] pour faire boire ceux qui porterent le corps de monseigneur saint Mamert et autres corps saints...

${ }^{44}$ Une vignette de tête de chapitre de la Sainte Jeanne d'Arc de Georges Goyau (Paris-Orléans, 1920) montre les deux saints évêques encadrant une fantaisiste ville médiévale dans ses remparts. L'illustration de l'ouvrage où tout ce qui concerne Jeanne, statues, maisons et cérémonies est en photo et donc «moderne», s'oppose aux vignettes, charmantes mais «moyenâgeuses » et archaïque, comme le souvenir des patres patrice... 
quelle la cité sauvée par la Pucelle a laissé jusqu'à présent les monuments qui portent les noms d'Aignan et d'Euverte pourrait paraître néanmoins dans une vision pessimiste des choses une preuve de noire ingratitude filiale. On nous promet des travaux, il faut croire au retour des enfants prodigues. 